\title{
Review
}

\section{Advancements in AAV-mediated Gene Therapy for Pompe Disease}

\author{
S.M. Salabarria ${ }^{\mathrm{a}}$, J. Nair ${ }^{\mathrm{a}}$, N. Clement ${ }^{\mathrm{a}}$, B.K. Smith ${ }^{\mathrm{b}}$, N. Raben ${ }^{\mathrm{c}}$, D.D. Fuller ${ }^{\mathrm{b}}$, \\ B.J. Byrne ${ }^{\mathrm{a}, *}$ and M. Corti ${ }^{\mathrm{a}, *}$

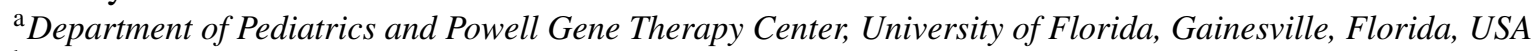 \\ ${ }^{\mathrm{b}}$ Department of Physical Therapy and Center for Respiratory Research and Rehabilitation, \\ University of Florida, Gainesville, Florida, USA \\ ${ }^{\mathrm{c}}$ Laboratory of Protein Trafficking and Organelle Biology, Cell and Developmental Biology Center, \\ National Heart, Lung and Blood Institute, NIH, Bethesda, Maryland, USA
}

\begin{abstract}
Pompe disease (glycogen storage disease type II) is caused by mutations in acid $\alpha$-glucosidase (GAA) resulting in lysosomal pathology and impairment of the muscular and cardio-pulmonary systems. Enzyme replacement therapy (ERT), the only approved therapy for Pompe disease, improves muscle function by reducing glycogen accumulation but this approach entails several limitations including a short drug half-life and an antibody response that results in reduced efficacy. To address these limitations, new treatments such as gene therapy are under development to increase the intrinsic ability of the affected cells to produce GAA. Key components to gene therapy strategies include the choice of vector, promoter, and the route of administration. The efficacy of gene therapy depends on the ability of the vector to drive gene expression in the target tissue and also on the recipient's immune tolerance to the transgene protein. In this review, we discuss the preclinical and clinical studies that are paving the way for the development of a gene therapy strategy for patients with early and late onset Pompe disease as well as some of the challenges for advancing gene therapy.
\end{abstract}

Keywords: Pompe disease, adeno-associated virus (AAV), lysosomes, autophagy, promoter, intravenous, intrathecal, intramuscular, immune-response, manufacturing

\section{INTRODUCTION}

\section{Pathophysiology of Pompe disease: A focus on neuropathology}

Pompe disease (glycogen storage disease type II) results from mutations in the gene encoding acid- $\alpha$-glucosidase $(G A A)$ which is responsible for lysosomal glycogen degradation. Lack of GAA activity results in pathologic glycogen accumula-

\footnotetext{
*Correspondence to: B.J. Byrne, MD, $\mathrm{PhD}$ and M. Corti, $\mathrm{PT}, \mathrm{PhD}$, University of Florida, Powell Gene Therapy Center, 1600 SW Archer Road, Gainesville, FL 32610, USA. E-mails: bbyrne@ufl.edu and m.corti@peds.ufl.edu.
}

tion creating deposits that ultimately disrupt cardiac, skeletal muscle, and central nervous system architecture and function $[1,2]$. The recent initiation of newborn screening for Pompe disease [3, 4] has raised estimates of prevalence to 1 in 8,700 [1]. Severity of the disease varies by age of onset and the rate of progression and is inversely correlated with residual GAA activity [5, 6]. Infantile-onset patients have GAA gene mutations causing absence of functional $G A A$. These patients are usually diagnosed during the first few months of life and if untreated, will soon die from cardiorespiratory failure [7]. Lateonset patients retain $<20 \%$ of normal GAA activity and show progressive motor dysfunction with res- 
piratory failure being the most common cause of mortality $[8,9]$. Severe respiratory insufficiency can occur without evidence for significant limb muscle weakness $[10,11]$. This feature is unique in Pompe disease compared to muscular dystrophies in which loss of ambulation precedes ventilatory insufficiency.

To date, there is no cure for Pompe Disease. Enzyme replacement therapy (ERT) was approved by the FDA in 2006 and is the only available treatment. This therapy involves bi-weekly infusion of recombinant human GAA $\left(\right.$ Myozyme $\left.^{\circledR}\right)$. Clinical trials show extended survival and some relief of the associated cardiomyopathy [12-18]. The ERT can also provide a transient, stabilizing impact on respiratory function, but many patients still progress to respiratory failure $[12,13,19,20]$. A particularly salient point is that ERT cannot be expected to effectively treat any existing neuropathology since the recombinant protein does not cross the blood-brainbarrier [21, 22]. Moreover, by temporarily mitigating cardiac and/or skeletal muscle problems, ERT can actually unmask a neurologic phenotype, particularly in infantile-onset patients [23]. The limited effectiveness of ERT, combined with established neuropathology in animal models [24-28] as well as in patients after failed ERT [2, 27] led to the hypothesis that neuropathology can substantially contribute to motor dysfunction in Pompe disease. Much of the evidence for neural dysfunction in Pompe disease, including early reports of neuropathology in patients [29-32] was summarized by Fuller at al. in 2013 [2]. Since then, more than 20 new publications have provided further evidence for neuropathology and neural impairment in Pompe disease. These new studies are summarized in Table 1 , and the reader is referred to earlier descriptions of central nervous system involvement in Pompe disease $[2,27]$. Collectively, the literature indicates that new treatment approaches capable of simultaneously treating muscle pathology and neuropathology are warranted.

Table 1

A summary of key reports from recent years that provide evidence of CNS or neuromuscular junction abnormalities in Pompe disease and relevant animal models

\begin{tabular}{|c|c|c|}
\hline Theme & Summary & Citations \\
\hline Motoneurons show pathology & $\begin{array}{l}\text { - } G A A^{-/-} \text {mouse shows prototypical motoneuron } \\
\text { histopathology in brainstem and spinal cord; hypoglossal and } \\
\text { phrenic motoneurons are earliest affected. } \\
\text { - } G A A^{-/-} \text {cord shows upregulation of apoptotic and } \\
\text { inflammatory genes } \\
\text { - Spinal cord from adult late-onset patient shows prototypical } \\
\text { motoneuron histopathology. }\end{array}$ & {$[24-26,83,95]$} \\
\hline Impaired neuromuscular junction & $\begin{array}{l}\text { - Late-onset patients have altered M-wave activation patterns } \\
\text { - } G A A^{-/-} \text {mouse has neuromuscular pathology including pre- } \\
\text { and post-synaptic changes } \\
\text { - } G A A^{-/-} \text {mouse has increased axonal fiber diameter and } \\
\text { demyelination in peripheral nerves. }\end{array}$ & {$[151-153]$} \\
\hline Vascular alterations & $\begin{array}{l}\text { - Radiological (MRI, CT) evaluation of late onset patients } \\
\text { indicates cerebral vasculopathy including dolichoectasia, } \\
\text { basilar artery fenestration and intracranial aneurysm. }\end{array}$ & [154-158] \\
\hline White and gray matter abnormalities & $\begin{array}{l}\text { - Early-onset patients show delayed myelination and pathology } \\
\text { in perivascular white matter. } \\
\text { - Late-onset patients have decreased connectivity in middle and } \\
\text { superior frontal gyrus, gray matter atrophy, and can show } \\
\text { impairments in the executive function }\end{array}$ & {$[154,159,160]$} \\
\hline Ventricular abnormalities & $\begin{array}{l}\text { - Early onset patients show ventricular enlargement, } \\
\text { cerebrospinal fluid accumulation, and periventricular white } \\
\text { matter abnormalities. }\end{array}$ & {$[160,161]$} \\
\hline CNS gene therapy & $\begin{array}{l}\text { - CNS targeted therapy using intraspinal, } \\
\text { intra-cerebroventricular, intravascular, intramuscular and } \\
\text { intrapleural delivery. } \\
\text { - Intravenous AAV.PHP.B-hGAA extends } G A A^{-/-} \text {mouse } \\
\text { lifespan } \\
\text { - Intramuscular AAV9-DES-GAA corrects neuromuscular } \\
\text { junction pathology. }\end{array}$ & {$[79,88,89,95,105,162]$} \\
\hline
\end{tabular}

Note that older citations are not included - rather the intent of this table to update the reader on recent publications. 
Lysosomes and autophagy in Pompe disease

Lysosomes are defined as membrane-bound organelles with acidic interior containing a collection of specific hydrolytic enzymes capable of breaking down a variety of macromolecules such as proteins, nucleic acids, lipids, and carbohydrates. A common hallmark of a large group of over 70 lysosomal storage diseases (LSDs) is the accumulation of undigested substrates within the lysosomal lumen, leading to lysosomal expansion [33]. For years, progressive disruption of this basic degradative function of the lysosome was considered an adequate explanation of the pathogenesis of LSDs, including Pompe disease - the first recognized storage disorder linked to the lysosome [34]. However, this long-held view of lysosomes as terminal degradation compartments is now a thing of the past. Instead, the lysosome is viewed as a sophisticated cellular center that controls a variety of cellular processes including cell growth, signaling, nutrient sensing, and autophagy [35, 36].

Macroautophagy (commonly referred to as autophagy) is a fundamental, evolutionarily ancient process that mediates the transfer of intracellular materials to lysosomes for degradation. The process involves the de novo formation of double-membrane vesicles, called autophagosomes, that sequester the cargo destined for degradation [37-40]. Autophagosomes fuse with lysosomes where the engulfed portion of cytoplasm is broken down and the resulting building blocks (e.g., amino acids, glucose, nucleotides, fatty acids) are exported back into the cytosol and utilized for energy generation and in biosynthetic pathways [41].

Initially, autophagy was described as a survival mechanism in response to cellular stressors, in particular amino acid starvation; induction of autophagy under nutrient-poor conditions allows the cell to derive new amino acids and energy from the random, non-selective (bulk) degradation of cellular components [42]. This response to environmental signals is mediated by the concerted actions of the mammalian target of rapamycin complex 1 (mTORC1), the master nutrient sensor and growth regulator, and AMP-activated protein kinase (AMPK), which is a key energy sensor. When nutrients are abundant, mTORC1 is recruited and activated at the lysosomal surface [43, 44]; once active, mTORC1 inhibits autophagy by phosphorylating autophagyinitiating kinase Ulk1. In contrast, when nutrients are insufficient, activated AMPK stimulates autophagy indirectly, by inhibiting mTORC1 (through phosphorylation of TSC2), and directly, by phosphorylating Ulk1 on distinct sites [45, 46]. Moreover, under nutritent-poor conditions, the inactive mTORC1 is detached from the lysosome and promotes autophagy by allowing translocation of transcription factors EB and E3 (TFEB and TFE3) to the nucleus where they activate genes involved in lysosomal and autophagosomal biogenesis [36, 47-50].

In addition to starvation-induced autophagy, autophagic machinery functions at low baseline levels to maintain cellular homeostasis by specifically recognizing and eliminating protein aggregates and damaged organelles [51, 52]. Based on the organelle destined for elimination, selective autophagy is called mitophagy (for mitochondria), lysophagy (for lysosomes), lipophagy (for lipid droplets), etc. Autophagic degradation of glycogen, a process termed glycophagy, was shown to have a critical importance in newborns [53-55].

Thus, the autophagy-lysosomal pathway plays a crucial role in the removal of worn-out organelles and toxic components as well as in cellular adaptation to various stresses and starvation. Dysfunctional autophagy has been associated with a range of pathologies including cancer, neurodegeneration, metabolic and cardiac diseases, and not surprisingly, LSDs including Pompe disease [56, 57]. The process is particularly important for the survival and stress adaptation of post-mitotic cells like neurons or muscle cells that are most affected in Pompe disease. Considering the evolving role of lysosomes, it is now amply clear that lysosomal dysfunction in the diseased muscle cells initiates a cascade of events far beyond the progressive glycogen accumulation. Disturbed autophagy and calcium homeostasis, oxidative stress and mitochondrial abnormalities, signaling and metabolic defects, all contribute to gradual muscle destruction in Pompe disease [58].

Autophagic defect in skeletal muscle of $G A A$ knockout mice (KO) [59] morphologically manifests as massive accumulation of cellular debris containing multivesicular bodies and lysosomes, broken lysosomal membranes, double-membrane autophagosomes with undigested cytosolic content, abnormal mitochondria, multilamellar bodies with electron-dense core, and glycogen particles both inside and outside vesicular structures [1]. The autophagic buildup can be visualized by electron microscopy of muscle biopsies and by confocal microscopy of isolated muscle fibers immunostained with lysosomal and autophagosomal markers. In fact, it is the latter 
technique that allowed us to appreciate the full extent of this pathology, which often spans the entire length of the fiber and occupies up to $40 \%$ of its volume in older $\mathrm{KO}$ mice $[60,61]$. In addition, undegraded autophagic substrates such as protein aggregates and p62/SQSTM1 can also be detected within the buildup areas by immunostaining. The routine analysis of muscle biopsies by Periodic-acid-Schiff staining might miss the buildup areas altogether, but upon closer look, one can often see "holes" in the core of muscle fibers on cross-sectional images (Fig. 1).

A similar picture is observed in muscle biopsies taken from Pompe disease patients, particularly those with late-onset form [60, 61]. The accumulation of undigested cellular debris [62,63] leads to the disruption of myofibrillar structures and a significant reduction in muscle force, likely exacerbated by the toxicity of accumulated substrates [64]. Experimental evidence indicates that autophagy in the diseased muscle is impaired at both the initial and final stages of the process: more autophagosomes are generated and less are fully resolved by fusion with lysosomes [65, 66]. This imbalance between the production and clearance of autophagosomes leads to progressive accumulation of autophagic debris. The failure to digest lysosomal glycogen to glucose may deprive muscle cells of energy source, thus initiating autophagic response to glucose starvation. Activation of AMPK-TSC pathway and inhibition of mTORC1 activity in cultured GAA-deficient myotubes and in muscle of $\mathrm{KO}$ mice support this hypothesis $[67,68]$. Alternatively, the diminished mTORC1

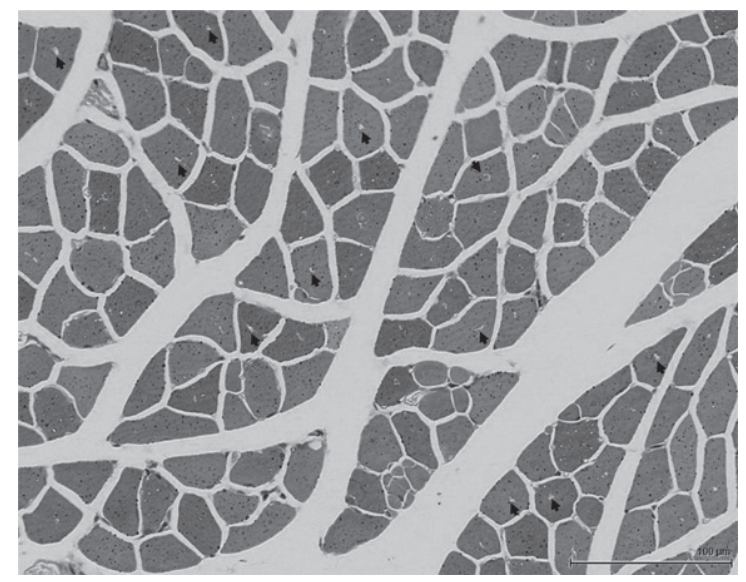

Fig. 1. PAS-stained sections (shown in black and white) of gastrocnemius muscle from a 7-month-old $G A A^{-/-}$mouse after 4 ERT injections. Arrows indicate "holes" in the core of muscle fibers. activity and ensuing induction of autophagy in the diseased muscle may be the initial insult originating from the inability of the damaged lysosomes to provide a proper platform for activation of this kinase.

Of particular importance is the data showing the link between defective autophagy and skeletal muscle resistance to ERT in Pompe disease. The autophagic buildup has a profound effect on vesicular trafficking and prevents efficient delivery of the therapeutic enzyme in muscle cells [65, 69]. Several attempts have been made to manipulate autophagy in muscle of KO mice by using genetic approaches: 1) genetic suppression of autophagy by inactivation of a critical autophagic gene, Atg7, in muscle of KO mice resulted in a significant reduction of lysosomal glycogen burden, thus serving as a substrate reduction therapy [70]; 2) overexpression of TFEB and TFE3 in GAA-deficient muscle cells and in muscle of $\mathrm{KO}$ mice stimulated autophagosomal-lysosomal fusion and exocytosis, reduced lysosomal size and glycogen content, and lessened the amount of cellular debris [65, 66]; 3) activation of mTORC1 in $\mathrm{KO}$ mice by knocking down its inhibitor, TSC2, largely rescued autophagic defect and reversed muscle atrophy [67, 68]. The common thread that runs throughout these experiments is that the currently available enzyme replacement therapy works well if skeletal muscle is cleared of autophagic buildup.

Importantly, recent data demonstrated that the reversal of autophagic pathology is feasible by using a more efficient next-generation drug for ERT. This new experimental drug, AT-GAA (Amicus Therapeutics) cleared muscle cells from lysosomal glycogen and reduced the number of myofibers with autophagic buildup in $\mathrm{KO}$ mice [71]. A similar reversal of autophagic accumulation in $\mathrm{KO}$ muscle has been recently achieved by gene therapy approaches [72, 73]. These results are, no doubt, very promising. It is, however, remains to be seen if the autophagic defect can be fully reversed and at what stage of the disease progression. In KO mice, the therapy was initiated when the animals showed little, if any, clinical manifestations of the disease. So far, there are no data showing the reversibility of this pathology in older symptomatic KO mice. This leaves open a possibility that the resolution of autophagic buildup in Pompe disease patients may require additional intervention. Of note, little is known about the potential role of neuronal autophagy in Pompe disease, although neuronal loss has been shown in animal models. 
Choice of Adeno-Associated virus (AAV) vector serotype, promoter and route of administration.

Pompe disease is a complex multi-systemic condition with manifestations in the cardiac, skeletal, and smooth muscles, endothelial cells, and motoneurons [12]. Therefore, targeting GAA expression in the desired target cells is an important aspect of gene therapy and depends on the selection of vector capsid serotype, specific promoter, and route of administration $[74,75]$.

In this regard, different strategies have been used to evaluate the efficacy of AAV-mediated GAA expression in animal models (Table 2). Many studies address the skeletal and cardiac manifestations of Pompe disease using an intravenous (IV) administration of AAV driven by ubiquitous promoters such as the human cytomegalovirus (CMV) or the hybrid CMV enhancer/chicken $\beta$-actin (CBA). Other studies have primarily focused on addressing the neurological manifestations of the disease using
CNS-targeted administration including intrathecal (IT), intra-cisterna (ICM) or intracerebroventricular (ICV) routes. Additionally, there is evidence that using AAV to drive liver expression of GAA under the control of liver-specifc promoters may be effective in ameliorating the peripheral manifestations in muscle. However, an important consideration is that the CNS penetration of GAA delivered via cross correction does not cross the blood brain barrier efficiently, much like the current ERT approach.

Aside from identifying the optimal route of administration for the primary tissues involved, choosing the correct promoter is a major step towards achieving successful therapeutic transgene expression. The CMV and CBA promoters provide rapid, robust and durable expression throughout the body except in the liver where these promoters may become inactive [76, 77]. On the other hand, muscle creatine kinase (CK) and hybrid $\alpha$-myosin heavy chain creatine kinase (MHCK7) promoters are primarily used to boost expression in skeletal and cardiac muscle while

Table 2

Preclinical and clinical studies in Pompe disease

\begin{tabular}{|c|c|c|c|c|}
\hline & $\begin{array}{c}\text { Route of } \\
\text { Administration }\end{array}$ & Serotype & Promoter & Citations \\
\hline \multirow[t]{24}{*}{ Preclinical studies } & IV & AAV2/8 & $\mathrm{CB}$ & {$[115]$} \\
\hline & & AAV2/8 & $\mathrm{CB}, \mathrm{LSP}$ & {$[91]$} \\
\hline & & AAV2/1, AAV2/8, AAV2/9 & CMV-lacZ & [87] \\
\hline & & AAV $2 / 2$, AAV $2 / 8$ & $\mathrm{CB}, \mathrm{LSP}$ & {$[165]$} \\
\hline & & AAV $2 / 8$ & LSP & {$[80]$} \\
\hline & & $\mathrm{AAV} 2 / 8$ & MHCK7 & {$[164]$} \\
\hline & & AAV8 & $\mathrm{CB}, \mathrm{LSP}$ & {$[122]$} \\
\hline & & $\mathrm{AAV} 2 / 9$ & $\mathrm{CB}$ & [116] \\
\hline & & $\mathrm{AAV} 2 / 9$ & DES & {$[86]$} \\
\hline & & $\mathrm{AAV} 2 / 8$ & MHCK7 & [163] \\
\hline & & AAV9 & DES, LSP & [124] \\
\hline & & AAV $2 / 8$ & LSP & [123] \\
\hline & & AAV8 & hAAT & [72] \\
\hline & & AAV $8 / 9$ & LiMP, LiNeuP & [81] \\
\hline & & AAV9, AAVB1 & DES & [89] \\
\hline & & AAV9 & $\mathrm{CMV}, \mathrm{CB}$ & [88] \\
\hline & IM, IV & AAV $2 / 2$, AAV $2 / 6$ & $\mathrm{CB}, \mathrm{MCK}$ & [92] \\
\hline & $\mathrm{IM}$ & AAV2, AAV1 & CMV & [93] \\
\hline & & AAV9 & CMV & [95] \\
\hline & & AAV9 & CMV & [162] \\
\hline & IP & AAV9 & DES & [79] \\
\hline & & AAV1 & CMV & [166] \\
\hline & IT or IS & AAV5 & $\mathrm{CB}$ & [167] \\
\hline & & AAV9, AAVrh10 & CAG & [103] \\
\hline \multirow[t]{4}{*}{ Clinical studies } & IM & AAV1 & $\mathrm{CMV}$ & {$[100,168]$} \\
\hline & & AAV9 & DES & NCT02240407 \\
\hline & & AAV1 & $\mathrm{CMV}$ & [145] \\
\hline & IV & AAV $2 / 8$ & LSP & NCT03533673 \\
\hline
\end{tabular}

Intravenous (IV); intramuscular (IM); intrapleural (IP); intrathecal (IT); intraspinal (IS); cytomegalovirus (CMV); CMV enhancer/ $\beta$-actin promoter (CB); liver specific promoter (LSP); liver-muscle promoter (LiMP); liver-neuron promoter (LiNeuP); desmin promoter (DES); muscle creatine kinase (MCK); hybrid $\alpha$-myosin heavy chain creatine kinase (MHCK7); human alpha 1-antitrypsin (hAAT); cytomegalovirus- $\beta$-galactosidase (CMV-lacZ); cytomegalovirus enhancer/ $\beta$-actin $\beta$-globin promoter (CAG). 
exhibiting low expression in non-muscle tissue and decreasing immune response to the transgene [78]. Other promoters include desmin (DES) which shows preferential expression in motor neurons, skeletal and cardiac muscles [77, 79], the neuro-targeted synapsin promoter, the liver-specific promoter (LSP) used to induce tolerance [80], and the liver-muscle promoter (LiMP) that provides expression in nondividing extra-hepatic tissues [81].

In the upcoming sections, we will discuss the routes of AAV administration that have been used to mitigate both the muscle and neuronal phenotypes in Pompe disease.

\section{Potential Gene Therapy approaches for Infantile Onset Pompe Disease (IOPD): Intravenous (IV) delivery}

ERT delays the onset of skeletal muscle weakness and the associated cardiomyopathy with minimal effects on the neurological aspects of the disease as GAA does not cross the blood brain barrier efficiently [82]. Systemic delivery of AAV vectors encoding GAA is an attractive alternative to ERT since cell autonomous correction in myocardium, skeletal muscle and the CNS will have significantly greater efficacy and comes with the potential for cross-correction of neighboring cells via GAA secretion. The need to correct the deficits in both the musculature and nervous system has become more apparent in recent years [83], and several recent preclinical studies have highlighted the feasibility of using rAAV-mediated IV gene delivery to correct the neuronal phenotype (Table 2).

In 2007, Mah et al. showed that a single systemic administration of an rAAV1 vector to restore GAA activity in vivo in a mouse model of Pompe disease resulted in long-term correction of the heart and skeletal muscle [84]. In addition, there was evidence of phenotypic correction of skeletal muscle exhibited through marked improvements in both soleus and diaphragm force mechanics. These results demonstrated the potential for systemic administration of an rAAV1 vector to mediate sustained therapeutic levels of correction in both skeletal and cardiac muscles [84]. Successful systemic administration using rAAV vectors to reduce glycogen content and augment cardiac, respiratory and motor neuron function in $G A A^{-/-}$mice was also demonstrated [85].

To compare and contrast the cardiorespiratory therapeutic potential of AAV vs. ERT, Falk et al. administered a single systemic injection of rAAV2/9DES-hGAA (AAV9-DES) or bi-monthly injections of recombinant human GAA (ERT) to $G A A^{-/-}$mice [86]. Three months post-treatment, there was an improvement in weight gain, elongation of the PR interval, increased ejection fraction, and a reduction in left ventricular mass suggesting cardiac correction for both the AAV- and ERT-treated groups. However, AAV9 treated mice showed increased GAA activity as well as reduced glycogen accumulation in cardiac and respiratory muscles, while ERT treated animals did not. Additionally, $G A A^{-/-}$mice given rAAV9DES had significant changes in respiratory function when compared to ERT-treated mice, suggesting partial correction of lower motor neurons and improved respiratory timing [86]. These findings are in accordance with the results reported by others [85, 87].

Lim et al. also demonstrated that IV administration of an rAAV9 variant, rAAV-PHP.B driven by an $\mathrm{MCH}$ promoter and expressing GAA, achieved therapeutic levels of GAA activity and reduced glycogen in both the myocardium and skeletal muscle. Furthermore, treated mice exhibited improved gait as well as very little to no peripheral neuropathy suggesting an effect on brain glycogen accumulation [88]. Similarly, Keeler et al. compared the systemic administration of a newly engineered vector with a high affinity for muscle and CNS, AAVB1-DES-hGAA to AAV9-DES-hGAA in 3-month-old $G A A^{-/-}$mice at the dose of $1 \times 10^{12} \mathrm{vg}$. AAVB1 treated animals displayed robust weight gains, near normal forelimb strength, and increased transduction in the diaphragm, tongue base, and the thoracic spinal cord when compared to AAV9. Both treated groups displayed above average GAA enzyme activity in the heart, diaphragm, tongue, gastrocnemius, and lung, and reduced glycogen accumulation in each of the muscles studied [89].

Although IV delivery is a successful strategy in mitigating the phenotype and underlying neuropathology of the disease, it faces crucial challenges regarding 1) cost and availability of high amount of product and 2) the host immune response against the vector capsid protein and transgene [90-92].

\section{Intramuscular-targeted GAA expression}

Preclinical studies in mice have demonstrated increased GAA activity and glycogen clearance following intramuscular injection in the gastrocnemius [75], the tibialis anterior [93], the heart [93], the diaphragm [94] and the tongue [95]. These pre- 
clinical studies strongly suggest that IOPD patients could benefit from a targeted intramuscular treatment. For example, the tongue appears to be particularly susceptible to impairments in Pompe disease, and animal studies show that the hypoglossal motoneurons which control the tongue are among the first cells to show histopathology and glycogen accumulation in the neonatal nervous system [95]. Children with Pompe disease receiving ERT have weak and ineffective swallowing [96] and speech disorders [13]. Neurological symptoms that include impaired language development, possibly indicating hypoglossal motor impairments, persist despite ERT [97]. LOPD patients also have lingual weakness, dysphagia and dysarthria despite ERT [98]. Pompe patients also have difficulty with saliva management, secretions and drooling [97]. Difficulties with phonation are also common in advanced Pompe disease [99]. Accordingly, "correction" of pathology in tongue motor units could have profound functional benefits in a clinical setting. A prior study in $G A A^{-/-}$mice indicates that intralingual treatment with rAAV-GAA has excellent potential for treating lingual dysfunction in Pompe disease. Specifically, a single intralingual injection of single stranded rAAV-GAA $\left(1 \times 10^{11} \mathrm{vg}\right)$ drove persistent GAA expression in both lingual myofibers and brainstem XII motoneurons. The GAA expression caused a reduction of glycogen accumulation with reversal of the histopathology.

The diaphragm has also been a muscle of interest for gene therapy delivery. Smith et al conducted a phase I/II clinical trial (NCT00976352) of intradiaphragmatic delivery of rAAV1-CMV-hGAA in five patients with IOPD. Each subject received a dose of either $1 \times 10^{12} \mathrm{vg}(n=3)$ or $5 \times 10^{12} \mathrm{vg}(n=2)$ of rAAV1hGAA and the patients were followed for 6 months post-treatment. Significant improvements were noted in each subject's unassisted tidal volume and length of time of unassisted breathing with median increases of $29 \%$ and $425 \%$, respectively [100]. A limitation of the intramuscular route is that the transduction is limited to the cells in the small area around the injection sites. A way to overcome this limitation is the application of multiple small volume intramuscular injections to increase transgene expression [101, 102].

\section{Potential gene therapy approaches for late onset Pompe disease (LOPD)}

\section{CNS-targeted GAA expression}

Since LOPD phenotype does not present significant cardiomyopathy [83], CNS-targeted gene delivery is more suitable for the treatment. This approach has the advantage of reducing the total exposure to the vector and, consequently, reducing the immune responses [83]. Further, CNS directed therapy will reduce the total required vector dose and consequently should reduce cost.

Direct administrations to the cerebrospinal fluid such as IT, ICM and ICV administrations have been used previously in efforts to deliver genes to the CNS. Hordeaux et al treated adult $G A A^{-/-}$ mice with a single ICM injection of AAV9-CAGhGAA or AAVrh10-CAG-hGAA [103]. Four months post-ICM dosing, glycogen buildup was completely cleared throughout the CNS including brain, cerebellum, brainstem, glial cells, myelin sheaths and the proximal and distal spinal cords in both treatment groups. However, the proportion of cervical and lumbar motor neurons reactive to PAS, with intracellular accumulation of glycogen, was lower in AAV9-treated vs. AAVrh10-treated mice. One-year post-dosing, the treatment led to improved motor coordination, increased global muscle strength, improved nerve conduction, and a reduction in hypertrophic cardiomyopathy.

An IT-targeted approach has been used in several clinical trials to date, but not for Pompe disease. These trials include a phase I safety study of IT of scAAV9/JeT-GAN for the treatment of giant axonal neuropathy (GAN) (NCT02362438), and three studies evaluating the safety and efficacy of nusinersen (ISIS 396443) administered by IT route to participants with infantile-onset Spinal Muscular Atrophy (SMA), later onset SMA, and presymptomatic SMA (NCT02193074/ NCT02292537 and NCT02462759). In December 2016, the Food and Drug Administration approved the antisense oligonucleotide Spiranza (nusinersen) for treatment of SMA [104].

Similarly, Lee et all tested the efficacy of ICV administration for the treatment of Pompe mice using synapsin I (Syn-I) promoter for neuron-specific expression (yfAAV9/3-Syn-I-hGAA; rAAVN-GAA). The authors compared the rAAV-treated animals with ERT-treated animals and untreated controls at 3, 6 and 9 months post-treatment. The rAAVN-GAA treated animals exhibited increased GAA activity and decreased glycogen content in the CNS but not in skeletal muscle or the liver. Additionally, rAAVN$G A A$-treated mice showed substantial improvement in motor coordination and balance with reduced astrogliosis and improved myelination; however, only minimal improvement in global muscle strength 
was observed, and no respiratory functional improvements occurred [105]. This study provides further evidence that the CNS pathology contributes to the burden of the disease while also demonstrates the inability of the neuron-specific promoter to fully attenuate motor problems as it does not target the skeletal muscles.

\section{Liver-directed GAA expression}

Using the liver as a target for gene therapy takes advantage of the high biodistribution of AAV vectors to the liver and also allows for lower doses of AAV. The liver is able to secrete high levels of many serum proteins and this is an advantage for high-level expression of GAA, especially when engineered to enhance secretion [81]. Additionally, liver-directed expression can lead to immune tolerance induction. The induction of immune tolerance has been a topic of much interest in the field of hemophilia and these approaches have been adapted to Pompe disease, including liver specific expression of a therapeutic gene [106, 107], blockade of coreceptors [108], and cell-transplantation of genetically modified cells [109].

In 2017, Puzzo et al administered low vector doses $\left(5 \times 10^{11} \mathrm{vg} / \mathrm{kg}\right.$ ) of rAAV8 containing a series of GAA transgenes optimized for hepatic expression to $G A A^{-/-}$mice [72]. This approach resulted in increased GAA secretion and uptake in respiratory and skeletal muscles. When the dose was increased to $2 \times 10^{12} \mathrm{vg} / \mathrm{kg}$, the attenuation of the disease phenotype was demonstrated in all muscles 3 months post-treatment. Additionally, the mice treated with either dose showed that the secreted GAA improved therapeutic efficacy and lower immunogenicity compared to non-engineered GAA.

In non-human primates (NHP), Puzzo et al. administered AAV8 vectors encoding the sp7- $\Delta 8$-coGAA transgene at a dose of $2 \times 10^{12} \mathrm{vg} / \mathrm{kg}$ [72]. Following administration, circulating GAA activity increased by 3- to 6-fold above baseline. In comparison to the controls, the animals injected with the sp7- $\Delta 8$ coGAA transgene had increased GAA enzyme levels in the heart, diaphgragm and other skeletal muscle thus demonstrating that the protein product was secreted into the circulation and taken up by the periphery [72]. This finding demonstrates the therapeutic potential of the AAV vector-mediated liver expression of secreted GAA to mitigate the disease phenotype in multiple tissues.

Similarly, Colella et al developed a hepatocyterestricted, AAV-mediated gene transfer to provide sustained transgene expression that in tandem prevents immune response to GAA [81]. They hypothesized the AAV delivery using cassettes controlled by liver-muscle (LiMP, ApoE-hAAT-C5.12) and liver-neuron (LiNeuP, ApoE-hAAT-hSYN) tandem promoters could express the transgene in both hepatic and extra-hepatic target tissues. Using two hGAA transgenes [72], $G A A^{-/-}$mice were treated with $2 \times 10^{12} \mathrm{vg} / \mathrm{kg}$ of AAV9 vector intravenously. Delivered in tandem, LiMP promoters drove liver and muscle transgene co-expression, while LiNeuP promoters drove co-epxression in hepatocyte and neuronal cells. Ultimately, Colella et al showed that LiMP and LiNeuP tandem promoters resulted in specific hGAA transgene co-transcription in the liver, muscle and CNS, respectively. Moreover, this approach prevented anti-hGAA immunity in $G A A^{-/-}$mice [81]. However, this approach is not suitable for pediatric patients. In fact, following AAV vector-mediated gene transfer, therapeutic efficacy can be lost as hepatocytes proliferate and the liver matures [72, 81].

Another liver specific approach was introduced by Koeberl and colleagues, who developed a strategy for immunomodulation with a low-dose AAV vector containing a liver-specific regulatory cassette (LSP) previously shown to induce tolerance [80]. In an attempt to induce immune tolerance to rhGAA and enhance the efficacy of ERT, the AAV-LSPhGAApA vector was given intravenously to $G A A^{-/-}$mice either before or after a dose of rhGAA. Administration of AAV-LSPhGAApA prolonged the survival of the treated groups regardless of whether the immune modulation was given before or after the initial rhGAA injection. Mice in the treated group had decreases in $\operatorname{IgG}, \operatorname{IgG} 1$, and $\operatorname{IgE}$ antibody titers by week 10 compared to the mock-treated mice [110]. In addition, regulatory $\mathrm{T}$ cells (Treg) were shown to contribute to immune tolerance in Pompe mice by aiding in desensitization to rhGAA [110-112]. Thus, targeted development of immune tolerance can increase the efficacy of ERT. This approach has the potential to enhance the efficacy of AAV vector-mediated gene transfer by preventing or suppressing the development of antibodies against the GAA transgene [80, 110].

In a follow-up study, Wang et al administered $1.6 \times 10^{13} \mathrm{vp} / \mathrm{kg}$ of $\mathrm{AAV} 2 / 8-\mathrm{LSPh} G A A \mathrm{pA}$ to $G A A^{-/-}$mice to evaluate the toxicity of the vector and to explore its use as an adjunct therapy to rhGAA ERT. Administration of the vector either before or after a single rhGAA injection followed by contin- 
uous ERT resulted in variable responses between male and female mice. Specifically, the vector treatment prevented an anaphylactic immune response in male $G A A^{-/-}$mice but not always in females. Two-weeks post dosing, vector treated males had increased GAA activity in the liver and diaphragm and treated males showed an increased GAA in the heart. The vector genome content was sustained in all tissues through 16-week post dosing for both males and females along with a sustained increase in GAA activity and decrease in glycogen accumulation. Overall, the safety of the AAV2/8-LSPhGAApA vector for liver-targeted gene therapy was, indeed, demonstrated; however, males exhibited a greater therapeutic efficacy in all tissues apart from the myocardium compared to females $[113,114]$.

In efforts to further investigate alternative immunemodulatory therapies, Han et al administered an anti-CD4 monoclonal antibody (mAb) intravenously prior to an injection of $1.0 \times 10^{11} \mathrm{vp}$ of pAAV$\mathrm{CBhGAApA}$ [115]. Following vector administration, anti-GAA IgG1 was quantified at 6 weeks along with glycogen and GAA content in the heart, diaphragm and skeletal muscle. In comparison to mice treated with the vector alone, the mice treated with the anti-CD4 mAb+vector had a substantial reduction of anti-GAA immuno- globulins, including $\operatorname{IgG} 1$, IgG2a, IgG2b, IgG2c, and IgG3. This effect was similar in mice treated with one vs. three injections of anti-CD4 mAb. Additionally, GAA activity was increased in the heart, diaphragm and liver of the anti-CD4 mAb-treated animals. As expected, there was a large reduction in glycogen content in the heart and liver following anti-CD4 $\mathrm{mAb}$ administration and only a slight reduction in the diaphragm and quadriceps. Pretreatment with anti-CD4 mAb also enhanced transduction efficiency in the liver as evidenced by the 6-fold increase in vector genome copy number. By administering anti-CD4 mAb, Han et al were able to attenuate the humoral immune responses to both vector and transgene, thereby increasing therapeutic efficacy [116]. Anti-CD4 administration prior to an AAV injection allows for a lower dose and avoids $\mathrm{T}$ cell responses to AAV capsid [85].

In the process of developing immune suppression and tolerance strategies, Koeberl's team noted that simply elevating GAA activity was not sufficient to completely clear accumulated glycogen in the muscle $[80,110,113,114,116]$. The authors hypothesized that a more efficient lysosomal trafficking of the enzyme may be needed for glycogen clearance in skeletal muscle. To address the issue, Han et al evaluated two combinatorial approaches. First, clenbuterol, a selective $\beta_{2}$-adrenergic receptor agonist, was used in combination with ERT to induce immune tolerance and increase the CI-MPR-mediated uptake of GAA [116-120]. This strategy increased CI-MPR expression in skeletal tissue, enhanced the uptake and trafficking of GAA to the lysosomes, and improved the efficacy of ERT. In the second approach, salmeterol, a selective $\beta_{2}$ agonist, was used to increase the efficacy of AAV mediated therapies when administered alongside AAV2/9-CBhGAApA [121]. Both strategies showed promise in enhancing GAA activity and glycogen clearance in skeletal muscle.

Franco et al developed an AAV8 vector containing the liver-specific promoter LSP (rAAV$\mathrm{LSPh} G A A \mathrm{pA}$ ) to drive hepatic hGAA expression and increase liver transduction [91]. Administration of rAAV-LSPhGAApA $\left(1 \times 10^{11}\right.$ or $\left.5 \times 10^{11} \mathrm{vg}\right)$ or rAAV-CBhGAApA $\left(1 \times 10^{11} \mathrm{vg}\right)$ to $G A A^{-/-}$mice resulted in 60-fold higher GAA expression in the rAAV-LSPhGAApA treated animals 12 weeks post dosing. Furthermore, rAAV-LSPhGAApA- treated animals had higher vector DNA in the heart and skeletal muscle and lower glycogen storage in striated muscles when compared to rAAV-CBhGAA-treated mice [91].

Han and colleagues also compared the efficacy of ERT against rAAV-LSP-GAA vector administration in mice. The assumption was that liver- specific expression of GAA with an rAAV vector expressing human GAA under the control of an LSP would suppress the antibody response and thereby increase therapeutic efficacy. Following a single injection of AAV2/8-LSPhGAA [122], there was no detectable antibody response. Moreover, the AAV8-GAA liver gene transfer was as effective as ERT. GAA activity was increased in the liver following both ERT and AAV administration, and glycogen content was reduced in both the myocardium and diaphragm. Following the identification of the minimum effective dose $\left(8 \times 10^{10} \mathrm{vg} / \mathrm{kg}\right)$, Hann et al confirmed that the administration of low dose AAV2/8-LSPhGAA prevents the humoral responses to GAA and that the liver expression of GAA provides cross-correction in distant organs by virtue of continuous secretion of GAA from the liver [123].

Another approach for simultaneously targeting the heart and CNS is the co-packaging of promoters. Using this approach, Doerfler et al. treated GAA-/mice intravenously with a copackaged AAV9 vector containing a liver specific promoter (LSP) and a codon optimized GAA (coGAA). Copackaging 
AAV9-LSP-coGAA did achieve persistent expression exclusively in the liver but failed to attenuate the Pompe disease pathology: neither of the two doses tested by Doerfler demonstrated therapeutic benefit in skeletal or cardiac muscle. In addition, with increased GAA activity in the liver, there was evidence of immune tolerance induction as the elicited immune response against GAA was minimal with no hepatotoxicity. As immune responses can be life threatening and can limit therapeutic efficacy, these findings emphasize the utility of liver directed gene therapy for attenuating immune responses [124].

\section{Challenges in Gene Therapy: Production \& Immune Response}

\section{Production of recombinant adeno-associated vectors for clinical application.}

The first AAV clinical trial specifically designed to treat Pompe disease was initiated in 2009 at the University of Florida [125]. Manufacturing of the clinical IND, rAAV1-CMV-hGAA, was completed at the Human Application Laboratory of the Powell Gene Therapy Center, University of Florida, using the standard co-transfection method in human embryonic kidney cells (HEK293) grown in monolayers in CellSTACKS $\left(\mathrm{CS}^{\circledR} 0^{\circledR}\right)$ [100, 126]. The functions required to package the recombinant genome were provided by a single helper plasmid harboring the wtAAV2 Rep, the wtAAV1 capsid proteins open reading frames (ORF), and the wtAdenovirus 5 helper functions. The vector genome was provided from a second plasmid harboring the expression cassette bracketed by wtAAV2 Invereted Terminal Repeats (ITRs) [126, 127]. Upon transfection of both plasmids into the producer cells, the virus was harvested from the cell pellets and purified by multiple chromatographic steps followed by a concentration and formulation step [126].

This production campaign generated $2 \mathrm{E}+14 \mathrm{vec}-$ tor genomes from approximately $5 \mathrm{O} \mathrm{CS} 10^{\circledR}$ and enabled clinical dosage of nine pediatric patients at doses of either $1 \times 10^{12}$ or $5 \times 10^{12}$ total $\mathrm{vg}$, for a total of $3.3 \times 10^{13} \mathrm{vg}$ from the clinical batch [100, 128]. Approximately $30 \%$ of the clinical production batch was used to perform supporting toxicology and bio-distribution studies, release testing of the clinical drug, and a stability study (Clément et al., unpublished). The stability of the clinical product was assessed over a 6-year period for infectivity, identity, purity and container integrity, and revealed no changes in the product over this extended time period.
It is worth noting that this first trial was designed for local delivery in the diaphragm at relatively low clinical doses.

An ongoing follow-up trial, initiated in 2016, addresses the impact of an immune modulation protocol in conjunction with the local delivery of AAV9-Des-hGAA in the tibialis anterior (TA) of adult Pompe patients, at a fixed dose of $4.6 \mathrm{E}+13$ vg. The manufacturing campaign, also conducted in at UF, HAL, used the same production method but with a modified therapeutic plasmid carrying a modified expression cassette, AAV-Des-hGAA, and a helper plasmid providing the capsid proteins for AAV serotype 9 (NCT02240407). Downstream processing was also modified to account for the difference in serotype-specific capsid biophysical properties. A total of $120 \mathrm{CS} 10^{\circledR}$ were processed for production of the final clinical batch, generating about $2 \mathrm{E}+15$ vg (Cleaver B.D., Clément N., et al; unpublished data). This enabled dosing of six adult patients (total $3 \mathrm{E}+14 \mathrm{vg}$ ), clinical release testing and the ongoing stability study. To date, no biological changes have been observed in the clinical batch up to 4 years post-manufacturing (Clément et al, unpublished).

While the transfection production platform in flat stocks was able to provide amount of clinical drugs sufficient to enable these two trials, the current need for muscular myopathies including Pompe disease, calls for vector amounts that are one to two order of magnitude higher to support both higher clinical doses as well as a systemic IV route of injection. The following example should better illustrate this challenge: the pre-clinical studies associated with the current and upcoming Pompe trials using AAV9Des-hGAA assessed immune modulation of an IV administration and utilized close to $1 \times 10^{16} \mathrm{vg}$; two entities teamed up to generate multiple test article lots over a 2-year period (UF PGTC and University of Pennsylvania Vector Core) [129] (NCT02240407), using several hundreds of cell culture multi-layer flasks.

A third Phase I/II trial is about to be initiated for Pompe disease with a therapeutic dose of $5 \mathrm{E}+13$ $\mathrm{vg} / \mathrm{kg}$ for systemic delivery in pediatric patients. The first cohort of 6 patients Phase I/II would require $5 \mathrm{E}+14$ to $1.5 \mathrm{E}+15 \mathrm{vg}$ per patient, or more than $1 \mathrm{E}+16$ $\mathrm{vg}$ for the trial. Based on previous yield this would require as much as $1000 \mathrm{CS} 10^{\circledR}$.

Transfection using adherent platforms has proven simply not feasible for AAV clinical manufacturing for scales exceeding $1-5 \mathrm{E}+10^{15} \mathrm{vg}$, and would not be suitable to support Phase III and BLA applications. 
Toward Large-scale manufacturing of clinical drug for Pompe disease gene therapy.

Over the past decade, research efforts have focused on the development of large-scale manufacturing processes for the production of pre-clinical and clinical batches of rAAV in order to meet the current demand. Both academic and industry laboratories have invested toward methods generating high yield producer cells $(>1 \mathrm{E}+5 \mathrm{vg}$ per cell) and/or highly scalable methods using suspension. To date, we can outline two major biological systems developed to serve this common goal as reviewed by Clement et al [126]: 1) Chemical Transfection of DNAs carrying the AAV and helper functions split over two or three plasmids; and 2) Viral Infection to deliver the DNA sequences to a host cell.

Chemical transfection is achieved by using either Calcium-phosphate $\mathrm{CaPO}_{4}$ or Polyethylenimine (PEI). The clear advantage of PEI is the increased overall yield per cell as well as its high performance in suspension format. Currently, $500 \mathrm{~L}$ bioreactors are routinely used in the industry settings to produce AAV [126]. The apparent simplicity of the system, mainly due to the availability of the raw materials (GMP-grade plasmids, cell banks and growth media), has made it one of the most popular platform. A version of this transfection approach was successfully implemented for the first AAV gene therapy commercial product approved for muscular dystrophy, spinal muscular dystrophy (SMA) (Zolgensma ${ }^{\circledR}$, Avexis-Novartis, 2019). HEK293 cells are grown as monolayers on the high surface iCellis bioreactor.

However, two major potential drawbacks face this method: the cost and availability of GMP grade plasmids, due to the high competing demand for immense amounts and the relatively limited number of manufacturing facilities. Another drawback is that the overall yield per cell is still relatively low as compared to infection-based methods, typically ranging in the low to high $E+04 \mathrm{vg} / \mathrm{cell}$ of purified product.

Infection-based platforms use a modified recombinant virus based on either the insect baculovirus (rBV) or Human Herpes Simplex Type I virus (rHSV) to deliver the AAV sequences to the producer cells. Both BV and HSV serve as helper for AAV replication and packaging and no further functions are required. Both systems work well in suspension format, using Sf9 for the BV system or BHK [130] or HEK293 for the HSV system [131]. A clear advantage of these two systems is the high yields generated both volumetrically and per cell. Typi- cally $>1 \mathrm{E}+5 \mathrm{vg} /$ cell or $>1 \mathrm{E}+14 \mathrm{vg} / \mathrm{L}$ are achieved with either system. Common challenges are the generation, screening and selection of the recombinant viruses and the production of viral banks prior to the production of AAV. The BV system has also suffered major drawbacks with the loss of AAV particle infectivity in some vector designs, as well as the persistent contamination of the insect cell lines with insect viruses [132]. The HSV system seems more versatile to produce highly infectious AAV irrespective of the serotypes. Both systems are currently being evaluated in ongoing clinical trials (UniQure, Voyager, AGTC, Solid Bio) and clinical data will help define both systems' strength and weaknesses.

A third platform combines both of the above with the introduction of the recombinant AAV genome and/or the AAV packaging functions Rep/Cap into the genome of the producer cell line to create a stable lines (PCL) that can produce rAAV upon rescue using a helper virus introduced by infection, typically Ad5 or Ad5 variant [126].

As described in Penaud-Budloo et al. [133], efforts in both developing assays to accurately detect the presence of process-derived and production-derived impurities, as well as improving downstream purification steps in order to reduce the load of impurities are ongoing and will be critical for the safety and efficacy of any manufacturing platform.

\section{Immune-responses to AAV capsid and the transgene, and vector re-administration}

A critical challenge for the success of gene therapy is the host immune responses to both the vector capsid and transgene product. These immune responses pose ongoing concerns regarding the safety, longevity, and extent of gene expression. Human immune responses to foreign antigens include humoral and cellular responses to the AAV capsid and to the transgene product. The humoral responses to AAV include activation of antigen-specific CD $4+\mathrm{T}$ helper cells $[134,135]$ and production of antibodies against the viral capsid [136]. Capsid neutralizing antibodies (NAbs) bind to the AAV capsid and may block or reduce the transduction of target cells. In humans, it has been shown consistently that administration of $\mathrm{AAV}$ in the dose range required for regional or systemic exposure leads to development of high and sustained anti-AAV antibodies [137-139]. For example, following IM administration of alipogene tiparvovec (Glybera, uniQure N.V.), all patients $(n=27)$ showed treatment-emergent anti- 
body responses to AAV1 [140]. Mouse, NHP, and human studies have shown that even low NAb titers can completely block transduction by AAV vectors [139, 141-143]. More recently, ongoing clinical programs for the treatment of neuromuscular diseases such as X-linked Myotubular Myopathy (XLMTM), Spinal Muscular Atrophy (SMA), Duchenne Muscular Dystrophy (DMD), Mucopolysaccharidosis (MPS), Giant axonal neuropathy (GAN) and others announced immune-responses to either the AAV capsid or the transgene with variable severity of safety signals. Management of the host's immune response to both the vector capsid and transgene product remains a critical challenge for the success of gene therapy.

In recent publications by Corti et al [144, 145], circulating anti-AAV1 and anti-GAA antibodies in a group of children with Pompe disease who received bilateral intra-diaphragmatic injection of rAAV1CMV-hGAA were studied. Three subjects receiving immunomodulation with rituximab (every 6 months) and Sirolimus (daily) to modulate immune reaction to ERT were compared to six subjects who did not receive immunomodulation. The group that did not receive immunomodulation demonstrated a 150fold increase in anti-AAV1 titer after exposure to AAV1. However, the three subjects, who received immunomodulation had no detectable levels of antiAAV1 or anti-GAA antibody through day 365 of the study $[144,145]$. In another study with a 2-year-old Canavan disease subject, immunomodulation with rituximab and sirolimus prevented formation of antiAAV9 antibodies and prolonged the detection of AAV vector DNA in the circulation for up to 17 months [146].

Another important challenge related to gene therapy is AAV re-administration. Patients may be restricted from clinical studies or commercial clinical use due to the development of a humoral immune response against the AAV capsid. Such exposure can occur by environmental exposure to wild type AAV, cross-reactivity of related AAV, or by sub-optimal therapeutic doses as part of early phase clinical trials. This is a critical unmet need in the gene therapy field as many subjects may require redosing later in life. Further, it is possible to achieve a more effective total dose by repeated lower doses of AAV over time. This approach would also increase the safety of the treatment by reducing the potential toxicity of a single high dose exposure. However, evidence suggests that, in the absence of immunomodulation, humoral and cellular memory responses to AAV may decrease duration of gene transfer and compromise subsequent use of the same vector [137-139].

Different strategies have been suggested to allow for vector re-administration. Our group has conducted preclinical studies in mice and NHPs demonstrating that immunomodulation strategies allow safe and effective repeat administration of the same AAV serotype (Corti Oral communication and [129]). To be able to use rituximab in mouse models, we crossed $G A A^{-/-}$mice with a line expressing the human CD20 (hCD20) antigen and generated hCD20 $+/ G A A^{-/-}$mice. Mice were pre-treated with rituximab for transient ablation of $B$ cells prior to AAV exposure and sirolimus for modulation of $\mathrm{T}$ cell response after dosing. Our results demonstrated that single and repeat administration of rAAV9-DES-hGAA was safe and that the use of this immune suppression regimen can prevent formation of antibodies against GAA and AAV9 capsid [129]. In a similar experiment using NHP, animals received single or repeated doses (four months apart) of rAAV9-DES-hGAA in the tibialis anterior (TA), with or without immunomodulation. Our data confirms that animals that received immunomodulation had higher GAA activity than those who did not receive immunomodulation.

Additionally, an ongoing clinical trial is testing repeat intramuscular (IM) administration of rAAV9-DES-GAA in adult Pompe patients (\#NCT02240407). This clinical study is a blinded crossover design to test safety and effectiveness of IM administration and re-administration of AAV9DES-hGAA into TA muscle using a similar immune modulation strategy. The immune modulation strategy is to ablate B cells using rituximab and sirolimus prior to the initial exposure to AAV9 in one leg and the subsequent exposure of the same vector to the contralateral leg after four months. Side of administration is randomized at first injection. Preliminary data from the first two patients enrolled confirm preclinical results showing that immunomodulation prevents antibody formation against AAV9 and the transgene (Corti Oral communication ASGCT $21^{\text {nd }}$ Annual Meeting; \#NCT02240407).

Our preclinical and clinical observations suggest that pharmacological immunomodulation prior and during AAV-mediated gene therapy is a successful strategy to modulate vector immunogenicity and to enable effective vector re-administration. Similar results were obtained by Meliani et al. [147] who demonstrated that when synthetic vaccine particles encapsulating rapamycin (SVP[Rapa]) are 
co-administered with AAV vectors, the anti-capsid humoral and cell mediated responses to AAV8-hF.IX and AAV8-GAA are reduced. This approach allowed successful vector re-administration in mice and NHP. However, this strategy would only work for liver gene transfer and not for our program, where we aim for a global gene transfer targeting both neurological and cardiac impairments. Other strategies have been proposed to address AAV re-administration and pre-immunity including plasmapheresis and capsid switching $[147,148]$. However, both of these strategies have some limitations that impact their clinical applicability. Plasmapheresis requires multiple sessions in a short period of time and it does not completely eliminate high titer antibody [149]. Capsid switching is influenced by high cross reactivity of anti-AAV antibody [150]. In addition, it adds additional complexity in AAV manufacturing and product characterization.

\section{CONCLUSIONS}

An effective treatment for Pompe disease remains an unmet medical need. However, in the last years, positive results from preclinical studies have increased the interest of several pharmaceutical companies towards gene therapy approaches. Targeting of CNS and muscle will be critical for successful therapy. Different gene therapy approaches have been considered in preclinical studies. It is unlikely that one gene therapy approach will address the different manifestations of Pompe disease, therefore, several gene therapy approaches will need to be considered based on the target population. In addition, strategies to manage large-scale production and immunogenicity should be considered for a successful therapy for the entire Pompe patient community.

\section{REFERENCES}

[1] Kohler L, Puertollano R, Raben N. Pompe Disease: From Basic Science to Therapy. Neurotherapeutics. 2018; 15(4):928-42.

[2] Fuller DD, et al. The respiratory neuromuscular system in Pompe disease. Respir Physiol Neurobiol. 2013;189(2):241-9.

[3] Prosser LA, et al. Using Decision Analysis to Support Newborn Screening Policy Decisions: A Case Study for Pompe Disease. MDM Policy Pract. 2018;3(1).

[4] Wasserstein MP, et al. The New York pilot newborn screening program for lysosomal storage diseases: Report of the First 65,000 Infants. Genet Med. 2019;21(3):631-40.

[5] Kroos M, et al. The genotype-phenotype correlation in Pompe disease. Am J Med Genet C Semin Med Genet. 2012;160C(1):59-68.
[6] Kroos MA, et al. Two extremes of the clinical spectrum of glycogen storage disease type II in one family: A matter of genotype. Hum Mutat. 1997;9(1):17-22.

[7] van den Hout HM, et al. The natural course of infantile Pompe's disease: 20 original cases compared with 133 cases from the literature. Pediatrics. 2003;112(2):332-40.

[8] Gungor D, et al. Survival and associated factors in 268 adults with Pompe disease prior to treatment with enzyme replacement therapy. Orphanet J Rare Dis. 2011;6:34.

[9] Hagemans ML, et al. Clinical manifestation and natural course of late-onset Pompe's disease in 54 Dutch patients. Brain. 2005;128(Pt 3):671-7.

[10] Pellegrini N, et al. Respiratory insufficiency and limb muscle weakness in adults with Pompe's disease. Eur Respir J. 2005;26(6):1024-31.

[11] Kansagra S, et al. Death from supine asphyxia in late onset pompe disease: Two patients. Am J Med Genet A. 2016;170(7):1928-9.

[12] Byrne BJ, et al. Pompe disease gene therapy. Hum Mol Genet. 2011;20(R1):R61-8.

[13] Byrne BJ, et al. Pompe disease: Design, methodology, and early findings from the Pompe Registry. Mol Genet Metab. 2011;103(1):1-11.

[14] van Capelle CI, et al. Effect of enzyme therapy in juvenile patients with Pompe disease: A three-year open-label study. Neuromuscul Disord. 2010;20(12):775-82.

[15] Jurecka A, et al. Effect of rapid cessation of enzyme replacement therapy: A report of 5 cases and a review of the literature. Mol Genet Metab. 2012;107(3):508-12.

[16] Toscano A, Schoser B. Enzyme replacement therapy in late-onset Pompe disease: A systematic literature review. J Neurol. 2013;260(4):951-9.

[17] Fernandez C, et al. Correction of a short cardiac PR interval in a 12-year-old girl with late-onset Pompe disease following enzyme replacement therapy. Genet Med. 2012;14(8):757-8.

[18] Chen CA, et al. Left ventricular geometry, global function, and dyssynchrony in infants and children with pompe cardiomyopathy undergoing enzyme replacement therapy. J Card Fail. 2011;17(11):930-6.

[19] van der Meijden JC, et al. Enzyme replacement therapy reduces the risk for wheelchair dependency in adult Pompe patients. Orphanet J Rare Dis. 2018;13(1):82.

[20] Yang CC, et al. Rapid progressive course of later-onset Pompe disease in Chinese patients. Mol Genet Metab. 2011;104(3):284-8

[21] Kikuchi T, et al. Clinical and metabolic correction of pompe disease by enzyme therapy in acid maltasedeficient quail. J Clin Invest. 1998;101(4):827-33.

[22] Raben N, et al. Enzyme replacement therapy in the mouse model of Pompe disease. Mol Genet Metab. 2003;80(12):159-69.

[23] Chan J, et al. The emerging phenotype of late-onset Pompe disease: A systematic literature review. Mol Genet Metab. 2017;120(3):163-72.

[24] Turner SMF, et al. Transcriptome assessment of the Pompe (Gaa-/-) mouse spinal cord indicates widespread neuropathology. Physiol Genomics. 2016;48(11):785-94.

[25] Turner SM, et al. Neuropathology in respiratory-related motoneurons in young Pompe $(\mathrm{Gaa}(-/-))$ mice. Respir Physiol Neurobiol. 2016;227:48-55.

[26] ElMallah MK, et al. Stimulation of Respiratory Motor Output and Ventilation in a Murine Model of Pompe Disease by Ampakines. Am J Respir Cell Mol Biol. 2015;53(3):326-35. 
[27] DeRuisseau LR, et al. Neural deficits contribute to respiratory insufficiency in Pompe disease. Proc Natl Acad Sci U S A. 2009;106(23):9419-24.

[28] Sidman RL, et al. Temporal neuropathologic and behavioral phenotype of 6 neo/6neo Pompe disease mice. J Neuropathol Exp Neurol. 2008;67(8):803-18.

[29] Gambetti P, DiMauro S, Baker L. Nervous system in Pompe's disease. Ultrastructure and biochemistry. J Neuropathol Exp Neurol. 1971;30(3):412-30.

[30] Mancall EL, Aponte GE, Berry RG. Pompe's Disease (Diffuse Glycogenosis) with Neuronal Storage. J Neuropathol Exp Neurol. 1965;24:85-96.

[31] Martin JJ, et al. Pompe's disease: An inborn lysosomal disorder with storage of glycogen. A study of brain and striated muscle. Acta Neuropathol. 1973;23(3):229-44.

[32] Martini C, et al. Intractable fever and cortical neuronal glycogen storage in glycogenosis type 2. Neurology. 2001;57(5):906-8

[33] Platt FM, et al. Lysosomal storage diseases. Nat Rev Dis Primers. 2018;4(1):27.

[34] Hers HG. alpha-Glucosidase deficiency in generalized glycogenstorage disease (Pompe's disease). Biochem J. 1963;86:11-6.

[35] Ballabio A. The awesome lysosome. EMBO Mol Med. 2016;8(2):73-6.

[36] Lawrence RE, Zoncu R. The lysosome as a cellular centre for signalling, metabolism and quality control. Nat Cell Biol. 2019;21(2):133-42.

[37] He C, Klionsky DJ. Regulation mechanisms and signaling pathways of autophagy. Annu Rev Genet. 2009;43: 67-93.

[38] Rubinsztein DC. Autophagy-alias self-eating-appetite and ageing. EMBO Rep. 2012;13(3):173-4.

[39] Rubinsztein DC, Codogno P, Levine B. Autophagy modulation as a potential therapeutic target for diverse diseases. Nat Rev Drug Discov. 2012;11(9):709-30.

[40] Rubinsztein DC, Shpilka T, Elazar Z. Mechanisms of autophagosome biogenesis. Curr Biol. 2012;22(1):R2934.

[41] Galluzzi L, Bravo-San Pedro JM, Kroemer G. Preface. Methods Enzymol. 2017;587:xxiii-xxix.

[42] Yang Z, Klionsky DJ. Mammalian autophagy: Core molecular machinery and signaling regulation. Curr Opin Cell Biol. 2010;22(2):124-31.

[43] Sancak Y, et al. The Rag GTPases bind raptor and mediate amino acid signaling to mTORC1. Science. 2008;320(5882):1496-501.

[44] Sancak Y, et al. Ragulator-Rag complex targets mTORC1 to the lysosomal surface and is necessary for its activation by amino acids. Cell. 2010;141(2):290-303.

[45] Kim J, et al. AMPK and mTOR regulate autophagy through direct phosphorylation of Ulk1. Nat Cell Biol. 2011;13(2):132-41.

[46] Egan D, et al. The autophagy initiating kinase ULK1 is regulated via opposing phosphorylation by AMPK and mTOR. Autophagy. 2011;7(6):643-4.

[47] Sardiello M, Ballabio A. Lysosomal enhancement: A CLEAR answer to cellular degradative needs. Cell Cycle. 2009;8(24):4021-2.

[48] Sardiello M, et al. A gene network regulating lysosomal biogenesis and function. Science. 2009;325(5939):473-7.

[49] Martina JA, et al. The nutrient-responsive transcription factor TFE3 promotes autophagy, lysosomal biogenesis, and clearance of cellular debris. Sci Signal. 2014;7(309): ra9.
[50] Puertollano R, et al. The complex relationship between TFEB transcription factor phosphorylation and subcellular localization. EMBO J. 2018;37(11).

[51] Perera RM, Zoncu R. The Lysosome as a Regulatory Hub. Annu Rev Cell Dev Biol. 2016;32:223-53.

[52] Lahiri V, Klionsky DJ. Spatially distinct pools of TORC1 balance protein homeostasis. Autophagy. 2019;15(4):5614.

[53] Kotoulas OB, Kalamidas SA, Kondomerkos DJ. Glycogen autophagy. Microsc Res Tech. 2004;64(1):10-20.

[54] Kotoulas OB, Kalamidas SA, Kondomerkos DJ. Glycogen autophagy in glucose homeostasis. Pathol Res Pract. 2006;202(9):631-8.

[55] Schiaffino S, Hanzlikova V. Autophagic degradation of glycogen in skeletal muscles of the newborn rat. J Cell Biol. 1972;52(1):41-51.

[56] Meng T, et al. Recent progress in the role of autophagy in neurological diseases. Cell Stress. 2019;3(5):141-61.

[57] Lieberman AP, et al. Autophagy in lysosomal storage disorders. Autophagy. 2012;8(5):719-30.

[58] Lim JA, et al. Pompe disease: Shared and unshared features of lysosomal storage disorders. Rare Dis. 2015;3(1):e1068978.

[59] Raben N, et al. Targeted disruption of the acid alphaglucosidase gene in mice causes an illness with critical features of both infantile and adult human glycogen storage disease type II. J Biol Chem. 1998;273(30):19086-92.

[60] Raben N, Roberts A, Plotz PH. Role of autophagy in the pathogenesis of Pompe disease. Acta Myol. 2007;26(1): 45-8.

[61] Raben N, et al. Deconstructing Pompe disease by analyzing single muscle fibers: To see a world in a grain of sand. Autophagy. 2007;3(6):546-52.

[62] Drost MR, et al. Effects of non-contractile inclusions on mechanical performance of skeletal muscle. J Biomech. 2005;38(5):1035-43.

[63] Ralston E, et al. Detection and imaging of non-contractile inclusions and sarcomeric anomalies in skeletal muscle by second harmonic generation combined with twophoton excited fluorescence. J Struct Biol. 2008;162(3): 500-8.

[64] Xu S, et al. Impaired organization and function of myofilaments in single muscle fibers from a mouse model of Pompe disease. J Appl Physiol (1985). 2010;108(5):13838.

[65] Spampanato C, et al. Transcription factor EB (TFEB) is a new therapeutic target for Pompe disease. EMBO Mol Med. 2013;5(5):691-706.

[66] Feeney EJ, et al. What else is in store for autophagy? Exocytosis of autolysosomes as a mechanism of TFEBmediated cellular clearance in Pompe disease. Autophagy. 2013;9(7):1117-8.

[67] Lim J, et al. mTORC1 Signaling is a Critical Regulator of Postnatal Tendon Development. Sci Rep. 2017;7(1): 17175.

[68] Lim JA, et al. Therapeutic Benefit of Autophagy Modulation in Pompe Disease. Mol Ther. 2018;26(7):1783-96.

[69] Nascimbeni AC, et al. Impaired autophagy affects acid alpha-glucosidase processing and enzyme replacement therapy efficacy in late-onset glycogen storage disease type II. Neuropathol Appl Neurobiol. 2015;41(5):672-5.

[70] Raben N, et al. Suppression of autophagy permits successful enzyme replacement therapy in a lysosomal storage disorder-murine Pompe disease. Autophagy. 2010;6(8): 1078-89. 
[71] Xu S, et al. Improved efficacy of a next-generation ERT in murine Pompe disease. JCI Insight. 2019;4(5).

[72] Puzzo F, et al. Rescue of Pompe disease in mice by AAV-mediated liver delivery of secretable acid alphaglucosidase. Sci Transl Med. 2017;9(418).

[73] McCall AL, et al. Reduction of Autophagic Accumulation in Pompe Disease Mouse Model Following Gene Therapy. Curr Gene Ther, 2019.

[74] Srivastava A, In vivo tissue-tropism of adeno-associated viral vectors. Curr Opin Virol. 2016;21:75-80.

[75] Sun B, Brooks ED, Koeberl DD. Preclinical Development of New Therapy for Glycogen Storage Diseases. Curr Gene Ther. 2015;15(4):338-47.

[76] Gray SJ, et al. Optimizing promoters for recombinant adeno-associated virus-mediated gene expression in the peripheral and central nervous system using selfcomplementary vectors. Hum Gene Ther. 2011;22(9): 1143-53.

[77] Pacak CA, et al. Tissue specific promoters improve specificity of AAV9 mediated transgene expression following intra-vascular gene delivery in neonatal mice. Genet Vaccines Ther. 2008;6:13.

[78] Salva MZ, et al. Design of tissue-specific regulatory cassettes for high-level rAAV-mediated expression in skeletal and cardiac muscle. Mol Ther. 2007;15(2):320-9.

[79] Falk DJ, et al. Intrapleural administration of AAV9 improves neural and cardiorespiratory function in Pompe disease. Mol Ther. 2013;21(9):1661-7.

[80] Sun B, et al. Enhanced response to enzyme replacement therapy in Pompe disease after the induction of immune tolerance. Am J Hum Genet. 2007;81(5):1042-9.

[81] Colella P, et al. AAV Gene Transfer with Tandem Promoter Design Prevents Anti-transgene Immunity and Provides Persistent Efficacy in Neonate Pompe Mice. Mol Ther Methods Clin Dev. 2019;12:85-101.

[82] Saraiva J, Nobre RJ, Pereira de Almeida L. Gene therapy for the CNS using AAVs: The impact of systemic delivery by AAV9. J Control Release. 2016;241:94-109.

[83] Byrne BJ, et al. Pompe disease gene therapy: Neural manifestations require consideration of CNS directed therapy. Annals of Translational Medicine, 2019.

[84] Mah C, et al. Physiological correction of Pompe disease by systemic delivery of adeno-associated virus serotype 1 vectors. Mol Ther. 2007;15(3):501-7.

[85] Sun B, et al. Correction of multiple striated muscles in murine Pompe disease through adeno-associated virus-mediated gene therapy. Mol Ther. 2008;16(8): 1366-71.

[86] Falk DJ, et al. Comparative impact of AAV and enzyme replacement therapy on respiratory and cardiac function in adult Pompe mice. Mol Ther Methods Clin Dev. 2015;2:15007.

[87] Pacak CA, et al. Recombinant adeno-associated virus serotype 9 leads to preferential cardiac transduction in vivo. Circ Res. 2006;99(4):e3-9.

[88] Lim JA, et al. Intravenous Injection of an AAV-PHP.B Vector Encoding Human Acid alpha-Glucosidase Rescues Both Muscle and CNS Defects in Murine Pompe Disease. Mol Ther Methods Clin Dev. 2019;12:233-45.

[89] Keeler AM, et al. Systemic Delivery of AAVB1-GAA Clears Glycogen and Prolongs Survival in a Mouse Model of Pompe Disease. Hum Gene Ther. 2019;30(1):57-68.

[90] Cresawn KO, et al. Impact of humoral immune response on distribution and efficacy of recombinant adenoassociated virus-derived acid alpha-glucosidase in a model of glycogen storage disease type II. Hum Gene Ther. 2005;16(1):68-80.

[91] Franco LM, et al. Evasion of immune responses to introduced human acid alpha-glucosidase by liver-restricted expression in glycogen storage disease type II. Mol Ther. 2005;12(5):876-84.

[92] Sun B, et al. Correction of glycogen storage disease type II by an adeno-associated virus vector containing a musclespecific promoter. Mol Ther. 2005;11(6):889-98.

[93] Fraites TJ, Jr., et al. Correction of the enzymatic and functional deficits in a model of Pompe disease using adeno-associated virus vectors. Mol Ther. 2002;5(5 Pt 1):571-8.

[94] Mah CS, et al. Gel-mediated delivery of AAV1 vectors corrects ventilatory function in Pompe mice with established disease. Mol Ther. 2010;18(3):502-10.

[95] Elmallah MK, et al. Sustained correction of motoneuron histopathology following intramuscular delivery of AAV in pompe mice. Mol Ther. 2014;22(4):702-12.

[96] van Gelder CM, et al. Facial-muscle weakness, speech disorders and dysphagia are common in patients with classic infantile Pompe disease treated with enzyme therapy. J Inherit Metab Dis. 2012;35(3):505-11.

[97] Jones HN, et al. Oropharyngeal dysphagia in infants and children with infantile Pompe disease. Dysphagia. 2010;25(4):277-83

[98] Dubrovsky A, et al. Expanding the phenotype of lateonset Pompe disease: Tongue weakness: A new clinical observation. Muscle Nerve. 2011;44(6):897-901.

[99] Hobson-Webb LD, Jones HN, Kishnani PS. Oropharyngeal dysphagia may occur in late-onset Pompe disease, implicating bulbar muscle involvement. Neuromuscul Disord. 2013;23(4):319-23.

[100] Smith BK, et al. Phase I/II trial of adeno-associated virusmediated alpha-glucosidase gene therapy to the diaphragm for chronic respiratory failure in Pompe disease: Initial safety and ventilatory outcomes. Hum Gene Ther. 2013;24(6):630-40.

[101] Greig JA, et al. Intramuscular injection of AAV8 in mice and macaques is associated with substantial hepatic targeting and transgene expression. PLoS One. 2014;9(11):e112268.

[102] Toromanoff A, et al. Safety and efficacy of regional intravenous (r.i.) versus intramuscular (i.m.) delivery of rAAV1 and rAAV8 to nonhuman primate skeletal muscle. Mol Ther. 2008;16(7):1291-9.

[103] Hordeaux J, et al. Long-term neurologic and cardiac correction by intrathecal gene therapy in Pompe disease. Acta Neuropathol Commun. 2017;5(1):66.

[104] Wurster CD, et al. Intrathecal administration of nusinersen in adolescent and adult SMA type 2 and 3 patients. J Neurol. 2019;266(1):183-94.

[105] Lee NC, et al. A Neuron-Specific Gene Therapy Relieves Motor Deficits in Pompe Disease Mice. Mol Neurobiol. 2018;55(6):5299-309.

[106] Hoffman BE, et al. Muscle as a target for supplementary factor IX gene transfer. Hum Gene Ther. 2007;18(7):60313.

[107] Mingozzi F, et al. Induction of immune tolerance to coagulation factor IX antigen by in vivo hepatic gene transfer. J Clin Invest. 2003;111(9):1347-56.

[108] Peng B, et al. Anti-CD3 antibodies modulate antifactor VIII immune responses in hemophilia A mice after factor VIII plasmid-mediated gene therapy. Blood. 2009;114(20):4373-82. 
[109] Doering CB, et al. Hematopoietic stem cells encoding porcine factor VIII induce pro-coagulant activity in hemophilia A mice with pre-existing factor VIII immunity. Mol Ther. 2007;15(6):1093-9.

[110] Sun B, et al. Immunomodulatory gene therapy prevents antibody formation and lethal hypersensitivity reactions in murine pompe disease. Mol Ther. 2010;18(2): 353-60.

[111] $\mathrm{Li} \mathrm{W}$, et al. Anti-CD25 mAb administration prevents spontaneous liver transplant tolerance. Transplant Proc. 2006;38(10):3207-8.

[112] van Wijk F, et al. CD4+CD25+T cells regulate the intensity of hypersensitivity responses to peanut, but are not decisive in the induction of oral sensitization. Clin Exp Allergy. 2007;37(4):572-81.

[113] Wang G, et al. Corrigendum to "Assessment of toxicity and biodistribution of recombinant AAV8 vector-mediated immunomodulatory gene therapy in mice with Pompe disease". Mol Ther Methods Clin Dev. 2015;2:15002.

[114] Wang G, et al. Assessment of toxicity and biodistribution of recombinant AAV8 vector-mediated immunomodulatory gene therapy in mice with Pompe disease. Mol Ther Methods Clin Dev. 2014;1:14018.

[115] Sun B, et al. Efficacy of an adeno-associated virus 8pseudotyped vector in glycogen storage disease type II. Mol Ther. 2005;11(1):57-65.

[116] Han SO, et al. Enhanced efficacy from gene therapy in Pompe disease using coreceptor blockade. Hum Gene Ther. 2015;26(1):26-35.

[117] Koeberl DD, et al. beta2 Agonists enhance the efficacy of simultaneous enzyme replacement therapy in murine Pompe disease. Mol Genet Metab. 2012;105(2): 221-7.

[118] Koeberl DD, et al. Enhanced efficacy of enzyme replacement therapy in Pompe disease through mannose6-phosphate receptor expression in skeletal muscle. Mol Genet Metab. 2011;103(2):107-12.

[119] Li S, et al. Adjunctive beta2-agonists reverse neuromuscular involvement in murine Pompe disease. FASEB J. 2013;27(1):34-44

[120] Farah BL, et al. Adjunctive beta2-agonist treatment reduces glycogen independently of receptor-mediated acid alpha-glucosidase uptake in the limb muscles of mice with Pompe disease. FASEB J. 2014;28(5):2272-80.

[121] Han SO, Li S, Koeberl DD. Salmeterol enhances the cardiac response to gene therapy in Pompe disease. Mol Genet Metab. 2016;118(1):35-40.

[122] Zhang P, et al. Immunodominant liver-specific expression suppresses transgene-directed immune responses in murine pompe disease. Hum Gene Ther. 2012;23(5):46072.

[123] Han SO, et al. Low-Dose Liver-Targeted Gene Therapy for Pompe Disease Enhances Therapeutic Efficacy of ERT via Immune Tolerance Induction. Mol Ther Methods Clin Dev. 2017;4:126-36.

[124] Doerfler PA, et al. Copackaged AAV9 Vectors Promote Simultaneous Immune Tolerance and Phenotypic Correction of Pompe Disease. Hum Gene Ther. 2016;27(1): 43-59.

[125] Byrne PI, et al. Phase I/II trial of diaphragm delivery of recombinant adeno-associated virus acid alphaglucosidase (rAAaV1-CMV-GAA) gene vector in patients with Pompe disease. Hum Gene Ther Clin Dev. 2014; 25(3):134-63.
[126] Clement N, Grieger JC. Manufacturing of recombinant adeno-associated viral vectors for clinical trials. Mol Ther Methods Clin Dev. 2016;3:16002.

[127] Clement N. Large-scale clinical manufacturing of AAV vector for systemic muscle gene therapy, in Muscle Gene Therapy, M. Dongsheng Duan and Jerry R Mendell, Editor. 2019, Springer.

[128] Smith BK, et al. Inspiratory muscle conditioning exercise and diaphragm gene therapy in Pompe disease: Clinical evidence of respiratory plasticity. Exp Neurol. 2017;287(Pt 2):216-24.

[129] Corti M, et al. Evaluation of Readministration of a Recombinant Adeno-Associated Virus Vector Expressing Acid Alpha-Glucosidase in Pompe Disease: Preclinical to Clinical Planning. Hum Gene Ther Clin Dev. 2015;26(3):185-93.

[130] Thomas DL, et al. Scalable recombinant adeno-associated virus production using recombinant herpes simplex virus type 1 coinfection of suspension-adapted mammalian cells. Hum Gene Ther. 2009;20(8):861-70.

[131] Adamson-Small L, et al. Sodium Chloride Enhances Recombinant Adeno-Associated Virus Production in a Serum-Free Suspension Manufacturing Platform Using the Herpes Simplex Virus System. Hum Gene Ther Methods. 2017;28(1):1-14.

[132] van Oers MM, Pijlman GP, Vlak JM. Thirty years of baculovirus-insect cell protein expression: From dark horse to mainstream technology. J Gen Virol. 2015;96(Pt 1):6-23.

[133] Penaud-Budloo M, et al. Pharmacology of Recombinant Adeno-associated Virus Production. Mol Ther Methods Clin Dev. 2018;8:166-80.

[134] Ertl HCJ. Preclinical models to assess the immunogenicity of AAV vectors. Cell Immunol, 2017.

[135] Ertl HCJ, High KA. Impact of AAV Capsid-Specific TCell Responses on Design and Outcome of Clinical Gene Transfer Trials with Recombinant Adeno-Associated Viral Vectors: An Evolving Controversy. Hum Gene Ther. 2017;28(4):328-37.

[136] Meliani A, et al. Determination of anti-adeno-associated virus vector neutralizing antibody titer with an in vitro reporter system. Hum Gene Ther Methods. 2015;26(2):4553.

[137] Mingozzi F, et al. Overcoming preexisting humoral immunity to AAV using capsid decoys. Sci Transl Med. 2013;5(194):194ra92.

[138] Mingozzi F, et al. Prevalence and pharmacological modulation of humoral immunity to AAV vectors in gene transfer to synovial tissue. Gene Ther. 2013;20(4):417-24.

[139] Mingozzi F, High KA. Immune responses to AAV vectors: Overcoming barriers to successful gene therapy. Blood. 2013;122(1):23-36.

[140] Ferreira V, et al. Immune responses to intramuscular administration of alipogene tiparvovec (AAV1LPL(S447X)) in a phase II clinical trial of lipoprotein lipase deficiency gene therapy. Hum Gene Ther. 2014; 25(3):180-8.

[141] Jiang H, et al. Effects of transient immunosuppression on adenoassociated, virus-mediated, liver-directed gene transfer in rhesus macaques and implications for human gene therapy. Blood. 2006;108(10):3321-8.

[142] Murphy SL, et al. Prolonged susceptibility to antibodymediated neutralization for adeno-associated vectors targeted to the liver. Mol Ther. 2008;16(1):138-45. 
[143] Manno CS, et al. Successful transduction of liver in hemophilia by AAV-Factor IX and limitations imposed by the host immune response. Nat Med. 2006;12(3):342-7.

[144] Corti M, et al. B-Cell Depletion is Protective Against AntiAAV Capsid Immune Response: A Human Subject Case Study. Mol Ther Methods Clin Dev. 2014;1.

[145] Corti M, et al. Safety of Intradiaphragmatic Delivery of Adeno-Associated Virus-Mediated Alpha-Glucosidase (rAAV1-CMV-hGAA) Gene Therapy in Children Affected by Pompe Disease. Hum Gene Ther Clin Dev. 2017;28(4):208-18.

[146] Corti MGD, Norman S, Coleman K, Liberati C, Elder M, Escolar M, Clement N, Clever B, Byrne B, Gao G. Immune Blockade in CNS Gene Therapy Improves Safety and Clinical Outcome. Molecular Therapy. 2018;26:32.

[147] Meliani A, et al. Antigen-selective modulation of AAV immunogenicity with tolerogenic rapamycin nanoparticles enables successful vector re-administration. Nat Commun. 2018;9(1):4098.

[148] Chicoine LG, et al. Plasmapheresis eliminates the negative impact of AAV antibodies on microdystrophin gene expression following vascular delivery. Mol Ther. 2014;22(2):338-47.

[149] Monteilhet V, et al. A 10 patient case report on the impact of plasmapheresis upon neutralizing factors against adenoassociated virus (AAV) types 1, 2, 6, and 8. Mol Ther. 2011;19(11):2084-91.

[150] Boutin S, et al. Prevalence of serum IgG and neutralizing factors against adeno-associated virus (AAV) types 1 , $2,5,6,8$, and 9 in the healthy population: Implications for gene therapy using AAV vectors. Hum Gene Ther. 2010;21(6):704-12.

[151] Falk DJ, et al. Peripheral nerve and neuromuscular junction pathology in Pompe disease. Hum Mol Genet. 2015;24(3):625-36.

[152] Corti M, et al. Altered activation of the tibialis anterior in individuals with Pompe disease: Implications for motor unit dysfunction. Muscle Nerve. 2015;51(6): 877-83.

[153] Wirsching A, Muller-Felber W, Schoser B. Are evoked potentials in patients with adult-onset pompe disease indicative of clinically relevant central nervous system involvement? J Clin Neurophysiol. 2014;31(4):362-6.

[154] Musumeci O, et al. Central nervous system involvement in late-onset Pompe disease: Clues from neuroimaging and neuropsychological analysis. Eur $\mathrm{J}$ Neurol. 2019;26(3):442-e35.
[155] Pichiecchio A, et al. Late-onset Pompe disease: A geneticradiological correlation on cerebral vascular anomalies. J Neurol. 2017;264(10):2110-8.

[156] Montagnese F, et al. Intracranial arterial abnormalities in patients with late onset Pompe disease (LOPD). J Inherit Metab Dis. 2016;39(3):391-8.

[157] Zhang B, et al. Late-onset Pompe disease with complicated intracranial aneurysm: A Chinese case report. Neuropsychiatr Dis Treat. 2016;12:713-7.

[158] Shah H, et al. Association of Low Lysosomal Enzymes Activity With Brain Arterial Dilatation. Stroke. 2018;49(8):1977-80.

[159] Ebbink BJ, et al. Classic infantile Pompe patients approaching adulthood: A cohort study on consequences for the brain. Dev Med Child Neurol. 2018;60(6):579-86.

[160] McIntosh PT, et al. Neuroimaging findings in infantile Pompe patients treated with enzyme replacement therapy. Mol Genet Metab. 2018;123(2):85-91.

[161] Parini R, et al. Long term clinical history of an Italian cohort of infantile onset Pompe disease treated with enzyme replacement therapy. Orphanet J Rare Dis. 2018;13(1):32.

[162] Todd AG, et al. Correcting Neuromuscular Deficits With Gene Therapy in Pompe Disease. Ann Neurol. 2015;78(2):222-34.

[163] Han SO, et al. Synergistic Efficacy from Gene Therapy with Coreceptor Blockade and a beta2-Agonist in Murine Pompe Disease. Hum Gene Ther. 2015;26(11):743-50.

[164] Sun B, et al. Antibody formation and mannose-6phosphate receptor expression impact the efficacy of muscle-specific transgene expression in murine Pompe disease. J Gene Med. 2010;12(11):881-91.

[165] Sun B, et al. Enhanced efficacy of an AAV vector encoding chimeric, highly secreted acid alpha-glucosidase in glycogen storage disease type II. Mol Ther. 2006;14(6):822-30.

[166] Conlon TJ, et al. Transfer of Therapeutic Genes into Fetal Rhesus Monkeys Using Recombinant AdenoAssociated Type I Viral Vectors. Hum Gene Ther Clin Dev. 2016;27(4):152-59.

[167] Qiu K, et al. Spinal delivery of AAV vector restores enzyme activity and increases ventilation in Pompe mice. Mol Ther. 2012;20(1):21-7.

[168] Byrne BJ, et al. Gene therapy approaches for lysosomal storage disease: Next-generation treatment. Hum Gene Ther. 2012;23(8):808-15. 